\title{
Extending Network life time by energy efficient data aggregation in WSN
}

\author{
Aaftab Alam ${ }^{1}$, Shish Ahmad ${ }^{2}$ \\ M..Tech Scholar ${ }^{1}$, Associate Professor ${ }^{2}$ \\ Department of Computer Science \& Engineering, Integral University, Lucknow, UP, India ${ }^{1,2}$ \\ Email: aftab9917@gmail.com ${ }^{l}$, shish@iul.ac.in ${ }^{2}$
}

\begin{abstract}
WSNs have attracted much attention during the recent two years and some commercial implementations such as environmental surveillance applications are being developed because of their many advantages such as limited size, minimal memory and energy requirements and good computation ability, as well as their cheap and dense deployment compared to fixed infrastructure wireless networks and even traditional ad hoc networks. As we know that sensor nodes are battery driven, we need to diminish energy consumption to utilize networks for extended duration, therefore it is required to diminish data traffic inside sensor networks, reduce amount of data that need to send to base station. The chief aspiration of this paper is to gather and aggregate data in an energy efficient manner so that network lifetime is enhanced. In this paper we focused on energy preservation of nodes by selecting the minimum path using Kruskal algorithm and aggregating the data by Huffman coding.
\end{abstract}

Keywords- Wireless sensor networks; data aggregation; energy preservation; WSN

\section{INTRODUCTION}

A wireless ad hoc network (WANET) is a temporary network that is set up between peer nodes to satisfy an immediate need. There are numerous protocols which are suitable for wireless ad-hoc networks, but are inappropriate for WSNs due to the distinctive needs of WSNs. Wireless sensor networks are different from ad-hoc networks in seven area, that is: node density, node proneness to failure, network size, frequency of topology changes, communication paradigm employed, resource limitations of nodes and node identification. A sensor network is generally deployed in a inaccessible or unreachable area, such as a tropical forest or a calamity area. In such circumstances the node proneness to failure is high due to the possibility of nodes being damaged and failing. Some nodes might also exhaust their energy resources quicker than other nodes due to being on a routing path that is utilized more than other paths. The communication paradigm employed in WSNs includes a large number of broadcasts that are sent through the network. These broadcasts are utilized for network set up and maintenance, discovery of neighbours and sending of data.

\subsection{Data Aggregation}

Sensor networks are collection of sensor nodes which co-operatively send sensed data to base station. As sensor nodes are battery driven, an proficient utilization of power is essential in order to utilize networks for extended period that's why it is needed to reduce data traffic inside sensor networks, diminish amount of data that is required to transmit to base station.

The procedure of accumulating the sensor data using aggregation techniques is known as Data aggregation. The working of common data aggregation technique is illustrated in the figure 1 . This technique utilizes the data from the sensor node and then aggregates the data by using some aggregation algorithms such as centralized method, LEACH (low energy adaptive clustering hierarchy), FTDA(Time Forecast Algorithm of Data Aggregation) etc. This aggregated data is transmitted to the sink node by choosing the competent path [1]. The entire process is illustrated in figure 1 .

\section{RELATED WORK}

One of the energy efficient routing protocols combining data fusion and routing is firstly provided by Low Energy Adaptive Clustering Hierarchy (LEACH) [2] [3] [4]. LEACH adopts a simple and efficient data fusion scheme, which fuses a large number of data message into a small set of meaningful information by compressing methods such as Beamforming [5]. Furthermore, LEACH selects clubs as the fundamental topology of the networks. Managing clubs does not need multi-hops and thus makes routing path straightforward. Nevertheless, Cluster Heads (CHs) are arbitrarily selected and clusters are formed by affiliating non- $\mathrm{CH}$ nodes to the 


\section{Available online at www.ijrat.org}

nearest $\mathrm{CH}$ based on arriving signal strength in LEACH. Consequently, average transmission distance from non- $\mathrm{CH}$ nodes to $\mathrm{CHs}$ is very large. Furthermore, $\mathrm{CHs}$ transmit data message unswervingly to $\mathrm{BS}$ deteriorate the energy dissipation of the wireless networks.

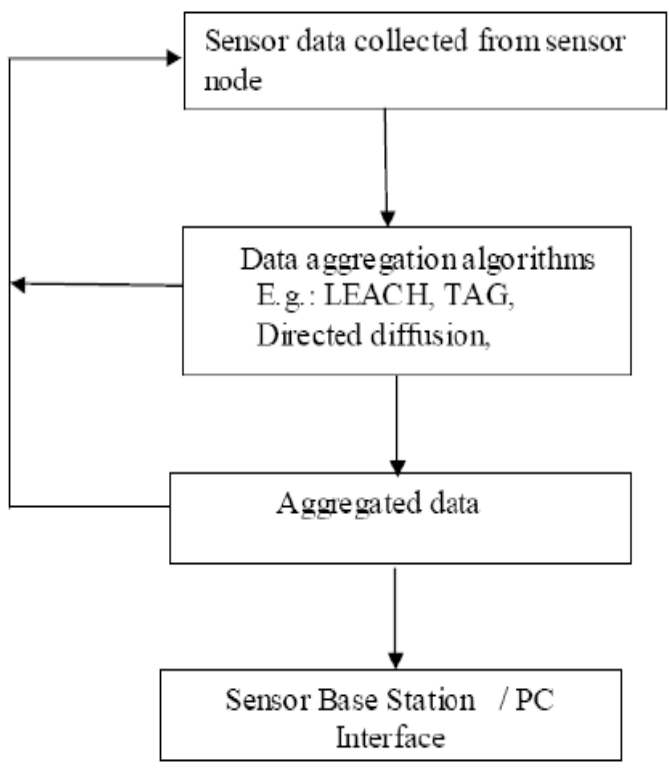

Fig 1: General architecture of the data aggregation algorithm

Mingxin Yang[6] proposed a data fusion algorithm FTDA based on the time prediction model in view of the problems of scalability of the prediction model and the ability to proactively identify data redundancy. The simulation results show that the algorithm can extend the network lifetime and save energy consumption.

Yanwei et. al. [7] proposed a power proficient wake up scheduling for data collection and aggregation. Here, TDMA is utilized as the MAC layer protocol and sensor nodes is scheduled with the consecutive time slots at diverse radio states while minimizing the number of state transitions.

Pengwei Hua et. al.[8] proposed an energy-proficient Adaptive Slice-based Secure Data Aggregation (ASSDA) method which can endorse the network performance under the restriction of node resource. This approach improves the effectiveness of data slicing, diminish the energy consumption of nodes, extend the network life time and maintain a highquality privacy preservation level in the same time. Theoretical analysis and simulation showed that ASSDA has superior performances in terms of privacy-preserving, communication overhead, node energy consumption and network lifetime.

\section{PROPOSED APPROACH KRSL}

\subsection{Methodology}

The proposed approach works in two stages.

1 Exploiting Kruskal algorithm for finding MST

2 Huffman coding approach is used for data compression.

The flow of our proposed approach is illustrated in figure 2 .

First, we apply the Kruskal algorithm on the network. Kruskal algorithm follows the minimum spanning tree concept. By Kruskal algorithm, we selected the minimum weight of the edge from one node to another node. After that, we make the tree which is chosen the minimum path of the node and all selected nodes of the network.

Huffman coding approach is used for data compression. In a networks data move one node to another node that time network has a lot of weight they consumed a lot of energy during data transmit one node to another node. When we apply Huffman coding for data compression, it reduces the network weight and also reduces the energy consumption

\subsection{Algorithm}

\section{Data structure used}

$\mathrm{S}=$ set of nodes

$\mathrm{E}=$ edges in the graph

$\mathrm{C}=$ cluster formed

$\mathrm{We}=$ weight of the edges $\mathrm{E}$

$\mathrm{w}=$ mean of the weight of the edges

$\sigma=$ standard Dividation of the weight of the edges

$\mathrm{M}=$ maximum size of cluster

$\mathrm{m}=$ minimum size of cluster

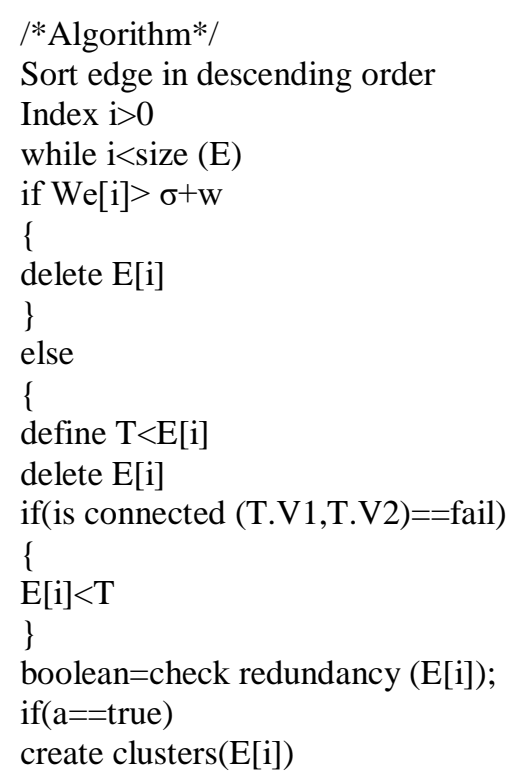


Available online at www.ijrat.org

check exit (E[i])

$\mathrm{i}->\mathrm{i}+1$

\}

check redundancy $(\mathrm{E}[\mathrm{i}])$

\{

define index->0

$\mathrm{C}[\mathrm{j}]=$ All nodes connected to $\mathrm{E}[\mathrm{i}] . \mathrm{V} 1$

$\mathrm{C}[\mathrm{j}+1]=\mathrm{All}$ nodes connected $\mathrm{E}[\mathrm{i}] . \mathrm{V} 2$

if $(\operatorname{size}(C[j])$ of size $(C[j+1])<m)$

return (false)

else

return(true)

\}

check exit(E[i])

\{

define index- $>0$

$\mathrm{C}[\mathrm{j}]=$ All nodes connected to $\mathrm{E}[\mathrm{i}] . \mathrm{V} 1$

$\mathrm{C}[\mathrm{j}+1]=$ All nodes connected to $\mathrm{E}[\mathrm{i}] . \mathrm{V} 2$

if $(\operatorname{size}(C[j])$ and $\operatorname{size}(C[j+1])<m)$

stop algorithm

else

continue

\}

\section{EXPERIMENTAL EVALUATION}

We used power disbursement model as shown in figure 3 .
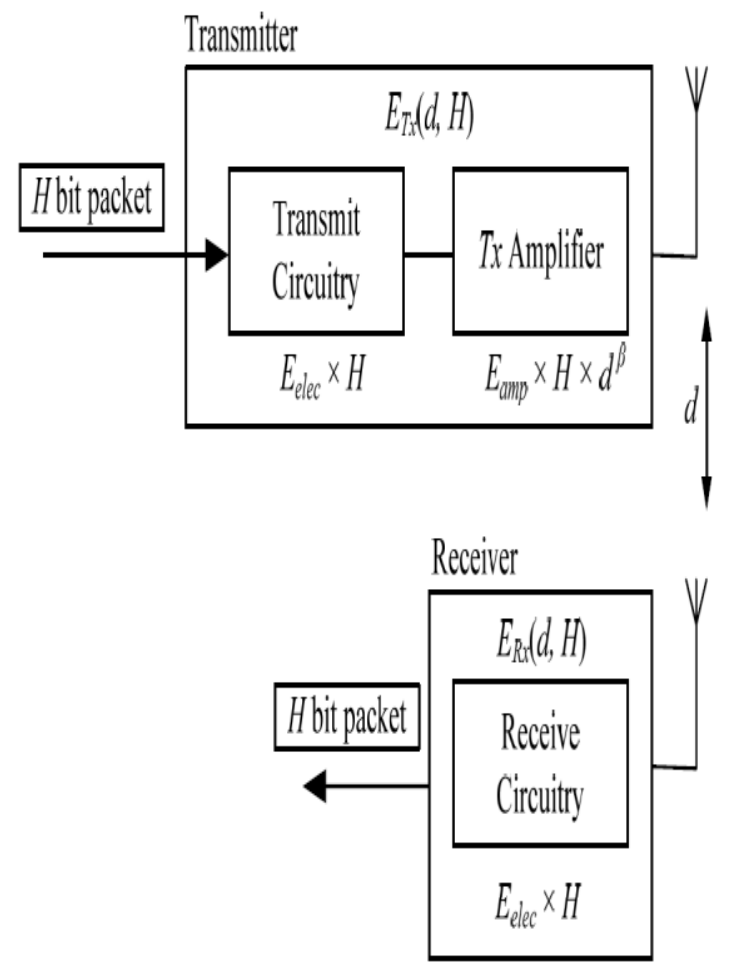

Fig. 3: Power disbursement model

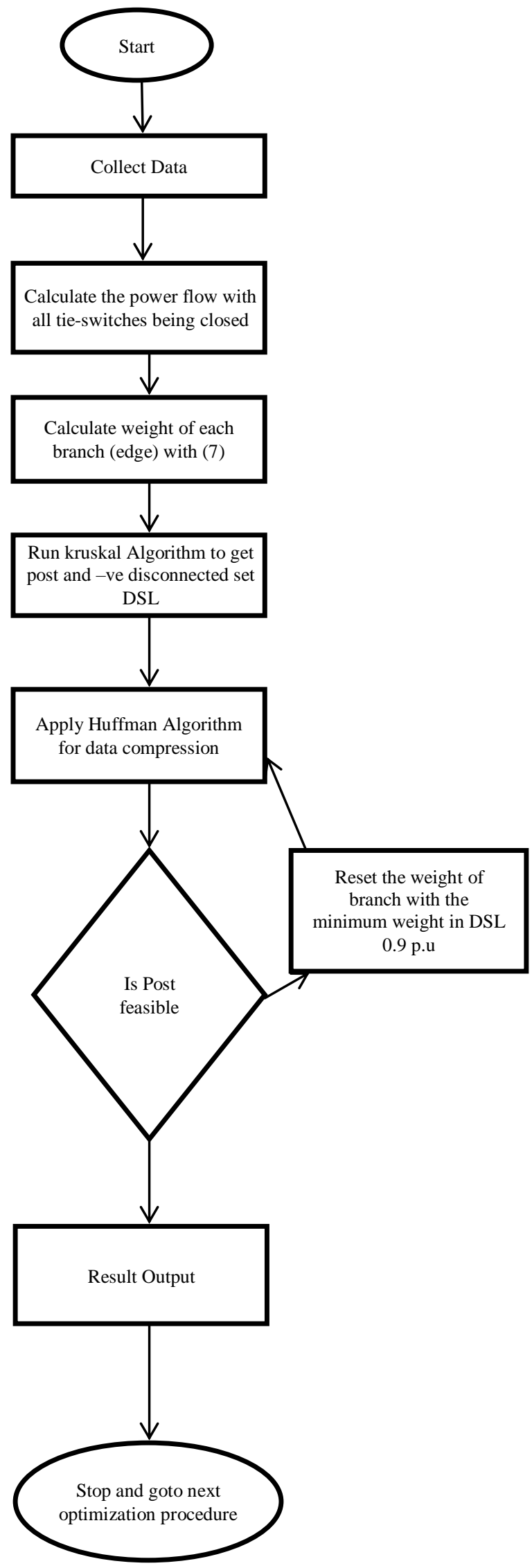

Fig 2: Flow of Proposed approach 


\section{Available online at www.ijrat.org}

In Figure 3, transmit circuitry or receive circuitry consumes $E_{\text {elec }}$ nano-joule energy in transmission or reception of per bit. Energy expenditure in the power amplification of each bit is depicted by $\mathrm{E}_{\mathrm{amp}}$. $\beta$ denotes the exponent for path loss. As a result, when a transmitter transmits a packet of $\mathrm{H}$-bit to the receiver, the overall energy expenditure can be determined as follows:

$$
\begin{aligned}
& E_{T x}(d, H)=E_{\text {elec }} \times H+E_{\text {amp }} \times H \times d^{\beta} \\
& E_{R x}(d, H)=E_{\text {elec }} \times H,
\end{aligned}
$$

We simulated our proposed protocol in a field with dimensions $500 \mathrm{~m} \times 500 \mathrm{~m}$ using $\mathrm{NS} 2$ with following parameters.

Table 1: Experimentation Parameters

\begin{tabular}{|l|l|}
\hline Parameter & values \\
\hline Area of monitoring field & $500 X 500 \mathrm{~m}^{2}$ \\
\hline Packet length & $32 \mathrm{bit}$ \\
\hline Initial energy & 12 jule \\
\hline Communication range & $120 \mathrm{~m}$ \\
\hline Sensing range & $500 \mathrm{~m}$ \\
\hline Transmitter electronic Etx & $50 \mathrm{~nJ} / \mathrm{bit}$ \\
\hline Receiver electronic Erx & $50 \mathrm{~nJ} / \mathrm{bit}$ \\
\hline $\begin{array}{l}\text { Energy of data aggregation } \\
\text { Eda }\end{array}$ & $6 \mathrm{nj} / \mathrm{bit} / \mathrm{signal}$ \\
\hline Free space factor & $6 \mathrm{pj} / \mathrm{bit} / \mathrm{m}^{2}$ \\
\hline Multi path factor & $0 ; 0013 / \mathrm{bit}^{2} \mathrm{~m}^{2}$ \\
\hline
\end{tabular}

The Snapshots of the implementations is shown in figure 4 .

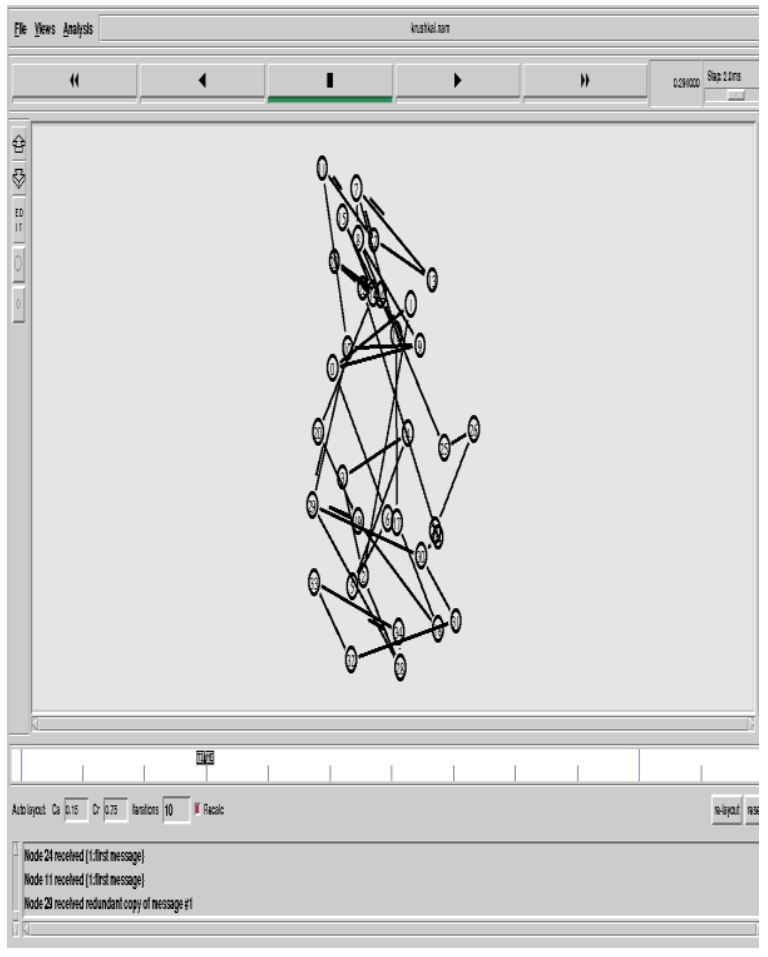

Fig. 4: Snapshots of the implementations

\section{RESULTS AND ANALYSIS}

Figure 5 shows the transmission time of the sensor. When the data transfer node to node that time make a cluster when the number of node increase and also increase cluster. One cluster has more than node some node repeat more than one cluster. So that time data transmission time increase

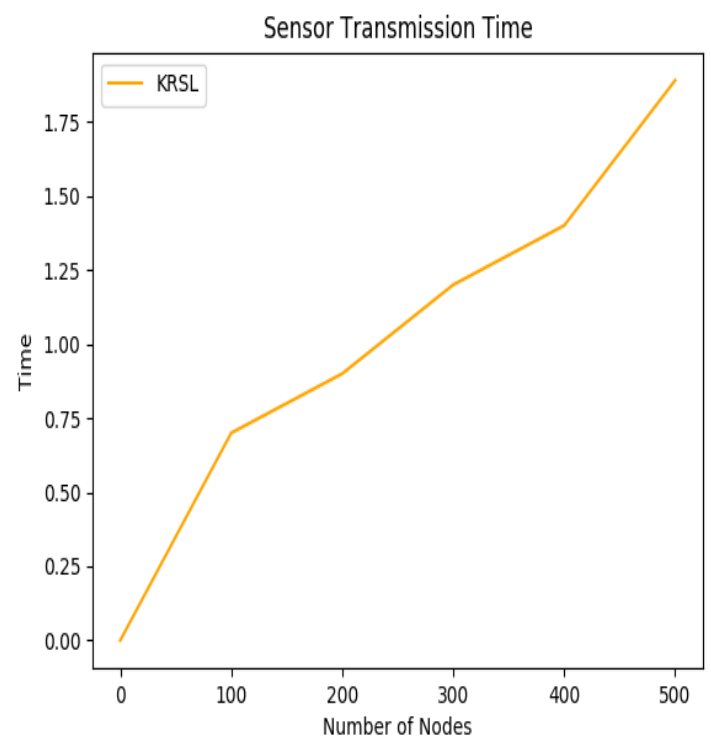

Fig. 5: Transmission time of the sensor 


\section{Available online at www.ijrat.org}

Figure 6 depicts the throughput which can be calculated as follows:

Throughput $=$ Number of the node which receives the data/number of the node which sends the data

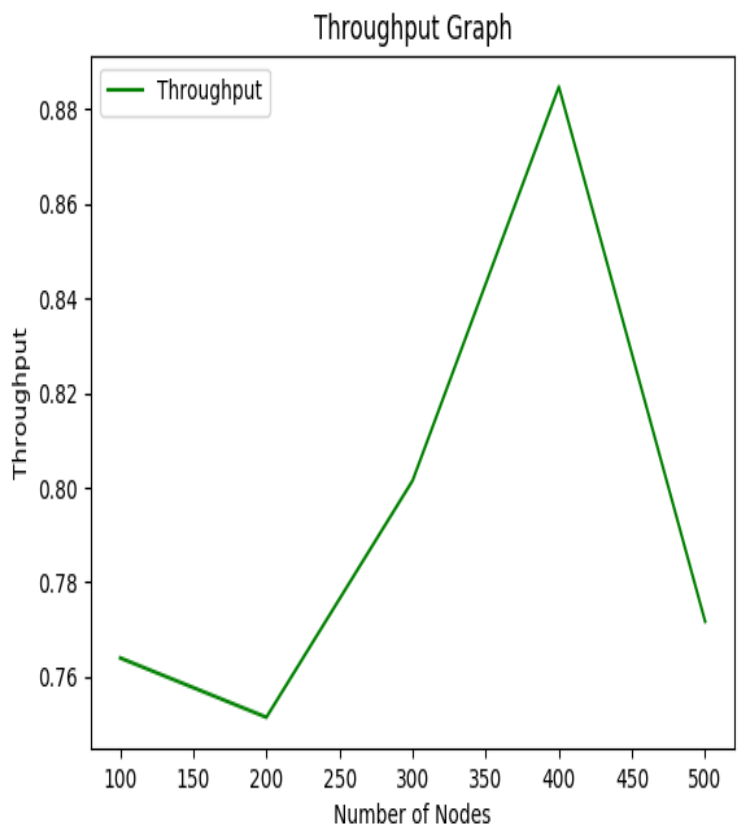

Fig. 6: Throughput of network

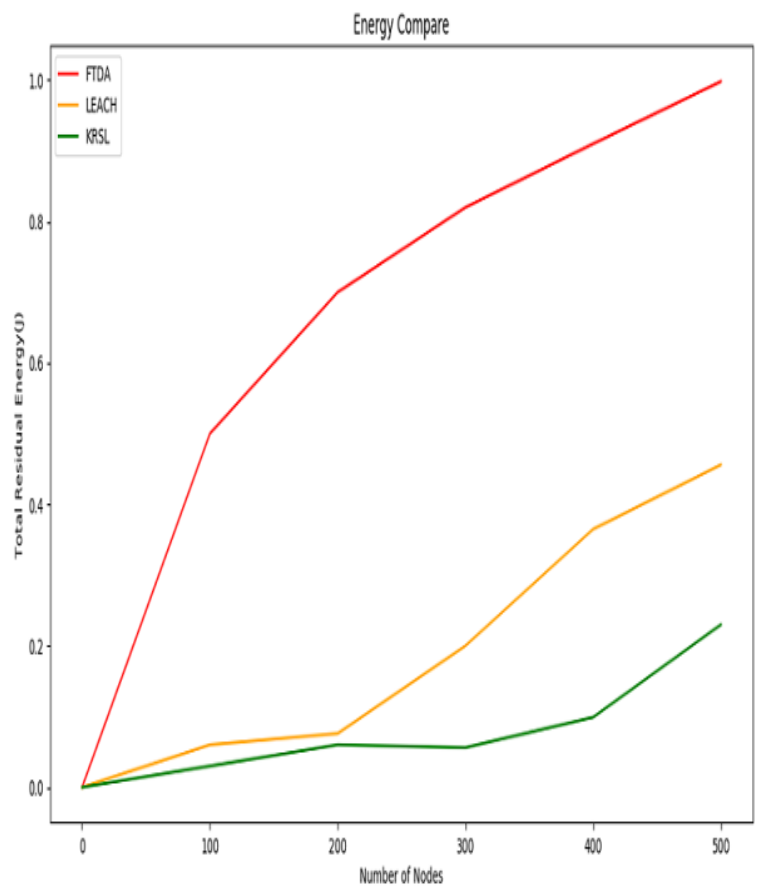

Fig. 7: Comparison of Energy consumption between proposed approach and FTDA and LEACH
Figure 7 shows that FTDA algorithm consumed a lot of energy when the number of nodes increasing continuously. Because FTDA algorithm repeats after 300 round that why FTDA consumed more energy according to a number of nodes. But the LEACH algorithm consumed less energy as compared to FTDA algorithm. Because LEACH algorithm is a progressive protocol in which most nodes transmit data to $\mathrm{CHs}$, and the clusters head aggregate and compress the data and forward it to the base station.

LEACH assumes that each node has a radio powerful enough to directly reach the base station or the nearest cluster head, but that using this radio at full power all the time would waste energy.

Now KRSL algorithm based on the cluster and it follows the minimum spanning tree concept. In this algorithm, all node selected based the minimum weight of the edges. Also, apply the Huffman coding algorithm for data compression. During data, the compression used some energy for data compression in the networks. Overall KRSL algorithm consumed less energy as compared to FTDA and LEACH algorithm.

\section{CONCLUSION}

Data aggregation is a necessary technology for wireless sensor networks for reducing the load and energy consumption through the network. In this paper, we presented a novel technique based Kruskal approach to build the spanning tree in wireless. The simulation result shows that the proposed KRSL algorithm consumes less energy as compared to FTDA and LEACH algorithm. Our algorithms try to minimize the number of redundant data packet transmissions by letting only a few nodes transmit more than once. The simulation results showed that our algorithms solve the data aggregation problem with a small number of packet transmissions.

\section{REFERENCES}

[1]. Patil NS, Patil PR. "Data aggregation in wireless sensor network", IEEE international conference on computational intelligence and computing research, pp. 28-29, 2010.

[2]. Fu, Chunyao, Zhifang Jiang, W. E. I. Wei, and Ang Wei. "An energy balanced algorithm of LEACH protocol in WSN." International Journal of Computer Science Issues (IJCSI), Vol. 10, no. 1, 354, 2013.

[3]. Malik, Meena, Yudhvir Singh, and Anshu Arora, "Analysis of LEACH protocol in wireless sensor networks." International Journal of Advanced Research in Computer Science and Software Engineering 3, no. 2 , 2013. 
International Journal of Research in Advent Technology, Vol.7, No.5, May 2019

E-ISSN: 2321-9637

Available online at www.ijrat.org

[4]. W. Heinzelman, A. Chandrakasan, and H. Balakrishnan. "Energy-Efficient Communication Protocols for Wireless Microsensor Networks". In Proceedings of Hawaiian International Conference on Systems Science, January 2000.

[5]. K. Yao, R. Hudson, C. Reed, D. Chen, and F. Lorenzelli, "Blind Beamforming on A Randomly Distributed Sensor Array System," in Proceedings of IEEE Workshop Signal Processing Systems, 1998.

[6]. Yang, Mingxin. "Data aggregation algorithm for wireless sensor network based on time prediction", IEEE 3rd Information Technology and Mechatronics Engineering Conference (ITOEC), pp. 863-867, 2017.

[7]. W. Yanwei, L. Xiang-Yang, L. YunHao, and L. Wei, "Energy-Efficient Wake-Up Scheduling for Data Collection and Aggregation," IEEE Transactions on Parallel and Distributed Systems, vol. 21, pp. 275-287, 2010

[8]. Hua P, Liu X, Yu J, Dang N, Zhang X. Energyefficient adaptive slice-based secure data aggregation scheme in WSN. Procedia Computer Science. 1.129.pp-18 